PANORAMA

ISSN: 1909-7433

ISSN: 2145-308X

ednorman@poligran.edu.co

Politécnico Grancolombiano

Colombia

\title{
INVESTIGACIÓN EN UNIVERSIDADES DE BOGOTÁ SOBRE EL CONOCIMIENTO Y DESTREZA DE LOS FUTUROS CREADORES DE CONTENIDO DIGITAL PARA LA CONSTRUCCION DE FORMATOS DE ANUNCIO EN FACEBOOK
}

Parra-Mayorga, Ricardo Andrés

INVESTIGACIÓN EN UNIVERSIDADES DE BOGOTÁ SOBRE EL CONOCIMIENTO Y DESTREZA DE

LOS FUTUROS CREADORES DE CONTENIDO DIGITAL PARA LA CONSTRUCCIÓN DE FORMATOS DE ANUNCIO EN FACEBOOK

PANORAMA, vol. 14, núm. 27, 2020

Politécnico Grancolombiano, Colombia

Disponible en: http://www.redalyc.org/articulo.oa?id=343964051006

DOI: https://doi.org/10.15765/pnrm.v14i27.1529

https://journal.poligran.edu.co/index.php/panorama/about/submissions\#copyrightNotice

Esta obra está bajo una Licencia Creative Commons Atribución-NoComercial-SinDerivar 4.0 Internacional. 
Artículos de investigación científica y tecnológica

PANORAMA, vol. 14, núm. 27, 2020

Politécnico Grancolombiano, Colombia

Recepción: 26 Julio 2019

Aprobación: 05 Julio 2020

DOI: https://doi.org/10.15765/ pnrm.v14i27.1529

Redalyc: http://www.redalyc.org/ articulo.oa?id=343964051006

https://journal.poligran.edu.co/

index.php/panorama/about/ submissions\# copyrightNotice CC BY-NC-ND
INVESTIGACIÓN EN UNIVERSIDADES DE BOGOTÁ SOBRE EL CONOCIMIENTO Y DESTREZA DE LOS FUTUROS CREADORES DE CONTENIDO DIGITAL PARA LA CONSTRUCCIÓN DE FORMATOS DE ANUNCIO EN FACEBOOK

\author{
RESEARCH AT UNIVERSITIES OF BOGOTÁ ON THE \\ KNOWLEDGE AND SKILL OF FUTURE CREATORS \\ OF DIGITAL CONTENT FOR THE CONSTRUCTION \\ OF ADVERTISING FORMATS ON FACEBOOK \\ PESQUISA NAS UNIVERSIDADES DE BOGOTÁ \\ SOBRE O CONHECIMENTO E HABILIDADE DE \\ FUTUROS CRIADORES DE CONTEÚDO DIGITAL \\ PARA A CONSTRUÇÃO DE FORMATOS DE \\ PUBLICIDADE NO FACEBOOK
}

Ricardo Andrés Parra-Mayorga raparramayorga@gmail.com Universidad de Bogotá Jorge Tadeo Lozano, Colombia

Resumen: Esta investigación tiene como propósito, en primera instancia, identificar cuáles son los formatos de anuncio permitidos por la plataforma social Facebook para generar interacción con las audiencias; posteriormente, evaluar el conocimiento y la destreza que tienen los estudiantes de últimos semestres en las carreras de Publicidad, Comunicación Social y Diseño gráfico en Bogotá, para desarrollar dichos formatos de anuncio de mensajes en Facebook. Finalmente, gracias a los resultados obtenidos, evidenciar oportunidades que permitan a los creadores de contenidos, adquirir habilidades de comunicación para plataformas sociales. Para el cumplimiento de este objetivo, se desarrolló un ejercicio de investigación a modo de diagnóstico exploratorio, bajo una metodología de recolección de datos cualitativa y cuantitativa, donde los participantes debían proponer una serie de contenidos en Facebook para una marca real. Dentro de los resultados se evidenció la necesidad de profundizar en temáticas como el conocimiento de los formatos e identificación de herramientas para la construcción de mensajes multimedia en Facebook, que les permita a los creadores de contenidos mejorar sus capacidades de transmisión de mensajes en la plataforma social de estudio. Esta investigación busca aportar al campo de la innovación tecnológica desde la perspectiva académica, donde se hace necesario dotar de herramientas a los futuros creadores de contenidos, que les permita ser profesionales competitivos en el área publicitaria-digital.

Palabras clave: Contenido digital, Facebook, anuncios, publicidad digital.

Abstract: The purpose of this research is, in the first instance, to identify which are the ad formats allowed by the social platform Facebook to generate interaction with 
audiences; subsequently, evaluate the knowledge and skills that students in their final semesters have in the careers of Advertising, Social Communication and Graphic Design in Bogotá, to develop such message ad formats on Facebook. Finally, thanks to the results obtained, show opportunities that allow content creators to acquire communication skills for social platforms. To fulfill this objective, a research exercise was developed as an exploratory diagnosis, under a qualitative and quantitative data collection methodology, where the participants had to propose a series of content on Facebook for a real brand. Among the results, the need to delve into topics such as knowledge of formats and identification of tools for the construction of multimedia messages on Facebook was evidenced, which allows content creators to improve their message transmission capabilities on the social platform study. This research seeks to contribute to the field of technological innovation from an academic perspective, where it is necessary to provide future content creators with tools that allow them to be competitive professionals in the advertising-digital area.

Keywords: Digital content, Facebook, advertisements, digital advertising.

Resumo: O objetivo desta pesquisa é, em um primeiro momento, identificar quais são os formatos de anúncios permitidos pela plataforma social do Facebook para gerar interação com os públicos; posteriormente, avaliar os conhecimentos e habilidades que os alunos dos últimos semestres possuem nas carreiras de Publicidade, Comunicação Social e Design Gráfico em Bogotá, para desenvolver esses formatos de anúncio de mensagem no Facebook. Por fim, graças aos resultados obtidos, mostram oportunidades que permitem aos criadores de conteúdo adquirir competências de comunicação para plataformas sociais. Para cumprir este objetivo, foi desenvolvido um exercício de investigação na forma de diagnóstico exploratório, sob uma metodologia de recolha de dados qualitativa e quantitativa, onde os participantes tiveram que propor um conjunto de conteúdos no Facebook para uma marca real. Entre os resultados, evidenciouse a necessidade de aprofundamento em temas como conhecimento de formatos e identificação de ferramentas para construção de mensagens multimídia no Facebook, o que permite aos criadores de conteúdo aprimorarem suas capacidades de transmissão de mensagens na plataforma social estude. Esta pesquisa visa contribuir para o campo da inovação tecnológica desde uma perspectiva acadêmica, onde é necessário dotar os futuros criadores de conteúdo de ferramentas que lhes permitam ser profissionais competitivos na área da publicidade digital.

Palavras-chave: Conteúdo digital, Facebook, anúncios, publicidade digital.

\section{INTRODUCCIÓN}

Internet ha tenido una evolución constante desde sus inicios como red pública hace más de veinte años. En la actualidad, se ha convertido en un medio adjunto al universo publicitario (Garrigo, 2006); dentro de este universo mencionado encontramos las plataformas sociales Facebook, Twitter, Instragram o YouTube, que son definidas como espacios virtuales donde se reúnen grupos de individuos relacionados por criterios o temáticas en común y que, en la actualidad, cumplen con un papel protagónico en el desarrollo de mensajes y posicionamiento de marca (Eduardo Norman-Acevedo, 2019). Por medio de internet, incluyendo las plataformas sociales, se puede llegar en el momento adecuado al consumidor real o potencial, siendo la publicidad interactiva la de mayor rentabilidad económica en comparación con medios tradicionales (Muela, 2008). De igual forma, los autores Sánchez, Schmidt, Zuntini y Obiol (2017, p.72), citan a Narayanan (2012), quien menciona: "dado la próspera tendencia de la utilización de Facebook, muchas organizaciones 
se han convertido en uno de los principales usuarios para sus estrategias de marketing en internet".

La compañía de tecnología HootSuite Media, Inc., en alianza con la agencia de marketing global "We are Social", revelaron en su informe anual de comportamiento de usuarios en internet, denominado DIGITAL IN 2018, que Facebook es la plataforma social más utilizada en el mundo, con un total de 2,17 mil millones de usuarios a comienzos de 2018 (Hootsuite, 2018, p.153). Esta cifra les ha permitido a las marcas identificar un nuevo espacio de comunicación con sus públicos, consumidores o seguidores para la transmisión de todo tipo de mensajes, incluyendo los comerciales o propagandísticos. Las marcas presentes en Facebook definen su participación por medio de páginas denominadas "fan page"; desde estos espacios se adhieren personas o fanáticos mediante la acción de seguimiento que permite ver de forma permanente las publicaciones que dichas marcas realicen en su perfil (Adán, Arancibia, López, Ramírez, Sospedra, \& Valladares, 2016, p.72). Los autores Mir, Fondevila y Gutiérrez (2006, p.29), citan a Payares (2012), quien menciona: la participación de las marcas en Facebook se hace mediante la "brand fan page", que da la opción a empresas, organizaciones, marcas o personas de mantener una presencia y comunicación profesional con sus audiencias en Facebook. Los autores Fernández y Díaz (2015), citan a la Interactive Advertising Bureau, IAB de España (2014), al referenciar el "Quinto estudio anual de Redes Sociales", donde se revela que para el 2014 las plataformas sociales en la población española habían logrado una penetración del $80 \%$; el $41 \%$ de los usuarios de redes sociales las emplea para seguir marcas, siendo Facebook la plataforma de mayor uso (E. Norman-Acevedo, 2018; Eduardo Norman-Acevedo, 2018).

Este contexto permite identificar una amplia oportunidad de investigación dirigida al desarrollo de mensajes en Facebook en favor de la construcción de imagen y reputación de una marca, producto, servicio, persona, o institución. La interacción de las compañías con sus fanáticos o seguidores en la plataforma social Facebook no solo se evidencia por medio de las fan pages, también se hace visible en la construcción de contenidos mediante piezas comunicativas denominadas "formatos de anuncio", categorizados de la siguiente manera: video, fotografía, imagen, enlace, nota de texto, artículo instantáneo, imagen 3D interactiva, live, slide show, experiencias instantáneas, carrusel, GIF, post 3D interactivo, foto 3D, live foto, eventos, anuncio de ofertas, anuncios de captación, historias, encuestas, fotografía $360^{\circ}$, imagen $360^{\circ}$ y video $360^{\circ}$ (Facebook Business, 2018). Identificar estas categorías es el punto de partida para definir una comunicación interactiva por medio de las plataformas sociales, con el fin de dar cumplimiento a los objetivos comunicativos de cualquier marca con sus públicos de interés (Velandia Vargas et al., 2019).

La presente investigación se desarrolla partiendo de la identificación de las nuevas herramientas (formatos de anuncio en Facebook), que nacen por la evolución de las plataformas sociales en internet, posteriormente se indaga sobre la destreza de los futuros creadores de contenidos, realizando un proceso de investigación a partir de la ejecución de 
un ejercicio que permitió evidenciar el desempeño de la población de estudio con las nuevas herramientas de comunicación. Finalmente, gracias a los resultados obtenidos, se espera promover y fomentar espacios educativos de fortalecimiento de competencias en el área digital para potenciar las cualidades de los estudiantes de las carreras de Publicidad, Comunicación social y Diseño gráfico de la ciudad de Bogotá, con respecto al conocimiento y desarrollo de contenidos interactivos.

\section{MARCO TEÓRICO}

Con la finalidad de presentar un esquema estructurado de investigación, se categorizan los componentes de la temática central a partir de cinco puntos fundamentales, que serán definidos y servirán para extraer las variables de evaluación del ejercicio que evidencia el contexto actual de los estudiantes o futuros creadores de contenidos, con respecto al desarrollo de mensajes interactivos para la red social Facebook.

\begin{tabular}{|c|l|}
\hline Categoría & \multicolumn{1}{|c|}{ Nombre de la categoría } \\
\hline a. & Publicidad digital y nueva publicidad digital \\
\hline b. & Redes sociales \\
\hline c. & Formatos de anuncio en Facebook \\
\hline d. & Interactividad \\
\hline e. & Futuros creadores de contenidos \\
\hline
\end{tabular}

Tabla 1.

Categorías de la temática de investigación. Fuente: elaboración propia, 2019.

\section{Publicidad digital y nueva publicidad digital.}

La publicidad digital hace parte de las estrategias definidas en el marketing digital, y se puede definir como todo tipo de acción comunicativa en favor del reconocimiento de una marca, y cuyo vehículo de transmisión esté ligado al desarrollo de mensajes pagos o gratuitos en internet. Las tácticas del marketing digital tienen características especiales y claramente diferenciadas de la promoción física: el entorno de trabajo es completamente distinto, pero, sobre todo, las posibilidades de diversificación, de análisis de resultados, de velocidad, de llegada al consumidor son enormemente superiores a las empleadas en otros medios (Moro \& Rodés, 2014, p.129). Victoria Carrillo y Ana Castillo (2005), afirman que existe una nueva publicidad digital, que se desarrolla por una metamorfosis gradual que viene sufriendo la publicidad desde un escenario de inconsistencia de formatos y estrategias, a una situación de mayor riqueza, valor formal y de contenidos; de igual forma, las autoras mencionadas también afirman que la publicidad digital hasta el momento es más o menos interactiva, en la medida en que es capaz de "prestar servicios", pero la nueva publicidad digital (NPD), debe combinar sin excusas las tres características de la interactividad: 
control activo, sincronía y multidireccionalidad (Carrillo \& Castillo, 2005, p.5). Las acciones de publicidad digital pueden o no causar un efecto positivo o negativo en las audiencias; por ello, las marcas - por medio del personal encargado de la transmisión de mensajestrabajan en el desarrollo de contenidos que logren empatía con el público buscando conexión, seguimiento y sentimiento positivo, en pocas palabras, generando engagement.

\section{Engagement como componente de la publicidad digital en redes sociales.}

Castelló, del Pino y Tur-Viñes (2015, p.125), mencionan que, en la actualidad, las marcas necesitan contar historias que van más allá de los atributos o ventajas competitivas de los productos y servicios; es allí donde se diseñan estrategias basadas en narraciones audiovisuales que buscan presentar la marca de forma cercana y transparente, conectando con la audiencia o generando engagement. Esta característica también es mencionada por los autores Ángel y Martínez (2014, p.279), quienes definen el engagement como la forma de "conectar emocionalmente" con un receptor por medio de contenidos creativos que promuevan experiencias y generen una implicación a través de los valores asociados al emisor del mensaje. Así mismo, las autoras Viñarás-Abad y CaerolsMateo (2016, p. 171), reseñan que el engagement se refiere a la capacidad que tienen las empresas u otros organismos de generar un vínculo o compromiso con la audiencia mediante sus acciones en la red, formando una comunidad que se identifique con la marca. A su turno, Valerio, Herrera, Villanueva, Herrera y Rodríguez, citan a Cvijikj y Michahelles (2013), quienes desarrollaron un marco conceptual en su investigación, proponiendo que el engagement digital en una red social depende de varios elementos: 1) el tipo de contenido que se publica, es decir, la naturaleza y el contenido del mensaje; 2) el formato de publicación, que puede ser entendido como el envoltorio del mensaje; y 3) el momento de publicación, que se refiere al tiempo cronológico o psicológico en el que ocurre una publicación.

\section{Redes sociales.}

Las plataformas sociales de internet le han permitido a la publicidad encontrar la oportunidad de explotar nuevas estrategias y acciones para la comunicación y el posicionamiento de mensajes. Para ampliar la definición de redes sociales en el ámbito digital, a continuación se presentan tres posturas que clarifican la razón de ser de estas plataformas que han revolucionado al mundo:

Redes sociales - Definición 1: un sitio de redes sociales es un servicio en línea en el que los miembros pueden establecer relaciones con base en amistad, afinidad, intereses comunes, ventaja comercial u otras razones. Una red social facilita estas interacciones al dejar que los miembros creen un perfil público o privado, especifiquen quién puede conectarse con ellos 
y compartan sus contenidos con los demás (Barker, Barker, Bormann, \& Neher, 2013, p.176).

Redes sociales - Definición 2: una red social es un sitio web multifuncional que permite a los usuarios con intereses personales conectarse entre sí para compartir información de todo tipo. Son plataformas con una gran carga viral que aprovechan muy bien el efecto boca a boca y que permiten a la empresa tener información útil sobre sus usuarios (Blanco, 2015, p.49).

Redes sociales - Definición 3: en 1998 se definió la red social de una forma genérica, como un conjunto de actores y de enlaces que los relacionan. Los actores (personas, organizaciones o cualquier otra entidad social), que se conectan por relaciones de amistad, parentesco, intercambio financiero o de información; es decir, por motivos sociales, cotidianos o profesionales (Carballal, 2012, p.71). Para De Salas (2010, p.82), las redes sociales aparecen como una oportunidad de impacto publicitario al autosegmentarse por intereses, aficiones, estilos de vida, entre otros. La popularidad de Facebook como plataforma de interacción ha promovido el desarrollo de grandes comunidades alrededor de marcas o productos, y así lo cita el libro "Marketing con Facebook" mencionando que en el año 2011 la marca Coca-Cola tenía en su fan page alrededor de 22 millones de seguidores (Zarrella \& Zarrella, 2011, p.19). Para Mir (2016, p.98), Facebook en los últimos años ha emergido como una poderosa plataforma de publicidad y, en comparación con otras redes sociales, representa la gran mayoría de actividad en la categoría de plataformas sociales (Barba Abad Magdalena; Alvarado Nando, Maritza; De La Rosa Daza, Deisy; Mora Ramírez, Ángela Julieta, 2019; DazaOrozco A.; Infante- Castillo E.G.; Norman-Acevedo, E.; RamírezVarela, F.; Arboleda-Muñoz, G.A.; Gil-Ángel, G.A.; Villada-Castillo, H.S.; Portela-Guarín, H.; García-Fernández, J.R.; Estévez-Ceballos,, F.E., C.E.; Cascante-Gatgens, 2019).

\section{Las redes sociales como parte de la publicidady el marketing.}

El marketing con redes sociales representa varias ventajas para las empresas, ya que les permite "encontrar talento, crear conciencia de la marca, encontrar nuevos clientes y ayudar a dirigir la inteligencia de marca y la investigación de mercado" (Barker, Barker, Bormann, \& Neher, 2013). Martínez y Sánchez (2011, p.475), enfatizan la importancia de vincular a las marcas con Facebook, al mencionar que los anunciantes utilizan los perfiles de redes sociales para entablar una comunicación personalizada entre la marca y el cliente, permitiendo conocer más que nunca a las audiencias, y facilitando así\# el ideal de la personalización de los contenidos y de la publicidad one to one. 


\section{Formatos de anuncio en Facebook.}

Facebook cuenta con espacios de orientación para los creadores de contenidos, donde hace especial énfasis en el uso de sus herramientas por medio de instrucciones que les permiten a los lectores de dicha información tener una definición clara de los instrumentos tecnológicos con los que cuenta la plataforma. Este es el caso de la "Guía para principiantes", donde Facebook Business (2018), define que es posible hacer uso de diversos formatos de anuncio en las plataformas de esta red social. La elección de cada uno de estos formatos depende del objetivo publicitario elegido y del conocimiento del anunciante quien tiene la decisión final sobre la elección del formato. Como se había mencionado anteriormente, Facebook cuenta con una amplia categoría de formatos aptos para el desarrollo de mensajes y que se mencionan a continuación: video, fotografía, imagen, enlace, nota de texto, artículo instantáneo, imagen 3D interactiva, live, slide show, experiencias instantáneas, carrusel, GIF, post 3D interactivo, foto 3D, live foto, eventos, anuncio de ofertas, anuncios de captación, historias, encuestas, fotografía $360^{\circ}$, imagen $360^{\circ}$ y video $360^{\circ}$ (Facebook Business, 2018).

\section{Interactividad.}

Para Carrillo y Castillo (2005), durante la navegación de los usuarios en espacios digitales no se debe entender la interactividad como el simple acto de dar clic sobre una parte o toda una pieza comunicativa; por el contrario, la interactividad debe ser mejor entendida desde la elaboración de contenidos que sean capaces de proporcionar "servicios" al usuario. De igual forma, los autores Romero y Fanjul (2010, p.129), afirman que la interactividad permite captar al consumidor, de manera que sea él mismo quien acuda a la red para informarse y entretenerse, estableciendo una conexión y haciéndolo participar de forma activa en el contenido publicitario. El desarrollo de contenidos de gran valor tiene la característica de proyectar en el receptor una percepción positiva sobre una marca, producto y, como lo menciona Rowles (2014), al afirmar: incluso cuando se trata de productos que normalmente generarían poco engagement, podemos estimularlo mediante un contenido interactivo y un buen branding digital.

$\mathrm{La}$ interactividad crea un puente entre los medios y los usuarios al permitir abordar ese espacio de relación entre ambas partes y analizar las diferentes instancias de selección, intervención y participación de los contenidos de un medio (Aguirre \& Odriozola, 2017, p.60). 177. vos perfiles publicitarios Siendo este panorama oleta. raciones pensando en las necesidades quePara Méndiz (2010, p. 40), la implantación de un esquema interactivo en el proceso publicitario ha desarrollado un cambio absoluto de paradigma comunicativo, mencionando que en dicho paradigma las características de los mensajes deben ser las siguientes: 1) comunicación bidireccional, 2) hibridación de publicidad e información y 3) experiencia lúdica. 
Cabe resaltar que la interactividad no solo logra brindar una experiencia innovadora y llamativa, también cumple un papel fundamental en la consecución de nuevos seguidores; así lo mencionan Arroyo, Calle, \& Van Wyk (2018, p.785), quienes definen que el aumento de seguidores en una fan page está ligado al desarrollo de la interacción con el receptor a partir de contenidos llamativos y de valor, que permitirán lograr objetivos digitales como desarrollo de engagement, posicionamiento de marca o reconocimiento de producto, servicio, entre otros.

Interactividad y realidad virtual.

Un complemento de la interactividad para la generación de engagement es la del desarrollo de contenidos de realidad virtual; de esta manera lo contextualizan Botella, García-Palacios, Baños y Quero (2007, p.18), quienes mencionan que la realidad virtual supone un cambio cualitativo con respecto a otras tecnologías, como la televisión o la pantalla de un ordenador, ya que permite una inmersión total en una simulación de la realidad, donde el usuario puede interactuar con el mundo virtual, de una forma similar a como interactúa con el mundo real.

\section{Futuros creadores de contenidos.}

Durante la etapa de desarrollo y maduración del marketing digital, las agencias publicitarias han tenido que generar reestructuraciones que incluyen nuevas posiciones y departamentos, siendo este panorama el que va a abonar el campo para dar nacimiento a nuevos perfiles publicitarios (Núñez, García, \& Abuín, 2013, p.177). Para Noguera, Martínez y Grandío (2011, p.16), autores de "Redes sociales para estudiantes de comunicación", las nuevas disciplinas desarrolladas a partir de la comunicación y publicidad digital necesitan de profesionales que comprendan la dinámica de la comunicación y la publicidad en internet. Es por ello por lo que dichos autores presentan cuatro perfiles laborales asociados con la publicidad digital: 1. Gestor de contenidos: profesional de la información y la documentación que define el tipo de contenidos. 2. Coordinador de marketing interactivo y relacional: encargado de determinar cuál es la mejor fórmula interactiva para que el mensaje llegue al consumidor. 3. Coordinador de marketing relacional: que puede prever el comportamiento de los clientes y llevar a cabo estrategias y promociones. 4.Responsable de comunidad o communitymanager: se encarga de construir, hacer crecer y gestionar comunidades alrededor de una marca o causa. Los estudiantes actuales de las carreras relacionadas con la profesión publicitaria tienen competencias de manejo de herramientas digitales al tener cercanía constante con estas, convirtiéndolos en opciones viables para el desempeño de labores comunicativas como la creación y administración de contenidos en internet, y así lo refiere Valerio (2015, p.52), al afirmar que los estudiantes universitarios de las nuevas generaciones tuvieron su primer contacto con el internet a edades tempranas. Por lo tanto, estos "nativos digitales" se 
sienten familiarizados y cómodos con el uso de la tecnología desde mucho antes de haber iniciado su formación universitaria.

En Bogotá existe una amplia cantidad de instituciones de educación superior universitaria profesional que ofrecen a los futuros creadores de contenidos, carreras ligadas al desarrollo del marketing digital como Publicidad, Comunicación social y Diseño gráfico, siendo estas profesiones las más relacionadas con el desarrollo laboral para la comunicación digital.

\section{MATERIALES Y METODOLOGÍA DE INVESTIGACIÓN}

Con el propósito de identificar el conocimiento y las habilidades que tienen los futuros creadores de contenidos con respecto al desarrollo de formatos de anuncio en Facebook, se realizó durante el año 2018 un estudio-diagnóstico investigativo que contó con la participación de estudiantes interesados en el campo profesional de la publicidad y el marketing digital en la ciudad de Bogotá. A continuación, se presenta una descripción de los materiales y la metodología de investigación implementada.

La presente investigación se clasifica de tipo exploratorio bajo una metodología mixta de recolección de datos (cualitativo y cuantitativo), aplicada a partir del desarrollo de un diagnóstico y la técnica de muestreo no probabilístico, mediante dos conglomerados definidos a criterio del investigador, partiendo del nivel de estrato socioeconómico de la siguiente manera: (conglomerado 1, individuos de estratos 1 y 2), (conglomerado 2, individuos de estratos 3,4 y 5 ).

- Descripción de la población a investigar: estudiantes hombres y mujeres de la ciudad de Bogotá, de semestres académicos quinto a décimo de las siguientes carreras profesionales: Comunicación social, Publicidad y Diseño gráfico, que tengan interés en el área del marketing digital y publicidad digital.

- Proceso de selección de la población: durante este proceso se realizó un listado de la totalidad de instituciones de educación superior en la ciudad de Bogotá que ofrecieran una de las siguientes carreras profesionales: Comunicación social, Publicidad y Diseño gráfico. Posteriormente se realizó una invitación formal para la participación en el ejercicio que busca evaluar la destreza de los estudiantes o futuros creadores de contenidos, con respecto al desarrollo de mensajes mediante los formatos de anuncio interactivos en Facebook. El número de universidades que participaron en la aplicación del ejercicio fue de siete instituciones. Se solicitó a cada institución participante contar con dos grupos de mínimo tres a máximo cinco estudiantes, de últimos semestres de las carreras mencionadas y que expresaran interés en el desarrollo profesional en el área de la publicidad digital. Adicionalmente, para participar en el ejercicio, fue necesario estar cursando o haber cursado una materia que tuviera relación directa con la construcción de productos o mensajes digitales multimedia y nuevos medios. 
- Muestra participante: 70 estudiantes.

- Variables de investigación: a partir del instrumento de recolección de la información, se desarrolló un análisis, partiendo de dos variables de investigación descritas a continuación:

1) Conocimiento de las herramientas: entendiendo esta definición como el nivel de conocimiento de los participantes con respecto a los formatos de anuncio en la red social Facebook.

2) Habilidad para el desarrollo de formatos de anuncio en Facebook: entendiendo esta definición como la capacidad para desarrollar formatos interactivos de anuncio en esta red social desde una perspectiva conceptual y estructural.

Diseño de la investigación, instrumentos de recopilación de la información.

- Ejercicio de diagnóstico: cada grupo participante en la investigación cumplió con un ejercicio comunicativo que consistió en el desarrollo de contenidos promocionales en la red social Facebook para una marca real. Durante el ejercicio se buscó evidenciar el conocimiento y la habilidad de desarrollo que tienen los estudiantes sobre los formatos de anuncio.

- Descripción de la marca utilizada para el ejercicio de investigación: en la ciudad de Bogotá se ubica la bolera Escape, un espacio recreativo y familiar que se caracteriza por contar con un ambiente decorativo alusivo al genero de música rock. Los espacios como paredes, mesas y demás elementos que intervienen en la bolera tienen una relación directa con bandas del género mencionado.

La bolera Escape tiene la necesidad de dar a conocer su mayor atributo entre el público bogotano: ser la única bolera rock del país, razón por la cual, para esta investigación, se hace necesario el desarrollo de una serie de acciones que incentiven la visita y renta del espacio a toda persona que se interese no solo en el juego de bolos, sino en experimentar este deporte acompañado de un ambiente musical.

Para efectos de la investigación, se utilizó la fan page de Facebook de la bolera Escape como desarrollo del ejercicio investigativo. Para ello, se construyó una plataforma web digital, donde los participantes de la investigación podían tener un contexto del ejercicio, así como fotografías y videos del espacio para el desarrollo de las ideas promocionales solicitadas. Dentro de la plataforma se desarrolló un brief textual y multimedia, con toda la información suficiente para la ideación creativa y resolución del problema propuesto a partir de acciones en las redes sociales de la bolera. Igualmente, la plataforma contó con formularios de texto para que los participantes describieran las ideas que tuvieran para promocionar la bolera Escape en la fan page de Facebook. Durante el desarrollo del ejercicio fue dispuesta una sala de sistemas en cada universidad. Los grupos participantes contaron con un computador con acceso a internet, para de esta forma tener acceso a la plataforma web donde recibieron el brief y describieron la solución propuesta.

Durante el ejercicio, se solicitó a cada grupo describir en el primer formulario de la plataforma un resumen textual sobre lo que el grupo 
consideraba que debería ser el mensaje y el concepto creativo a comunicar para el cumplimiento de los objetivos promocionales de la bolera en redes sociales. Posteriormente, el ejercicio les solicitaba a los grupos describir una parrilla de cinco contenidos multimedia (correspondientes a cinco días de la semana), basados en los formatos de anuncio (que conozcan) para Facebook, donde se busque llamar la atención y lograr interacción de las personas que tengan algún tipo de cercanía con la fan page de la bolera Escape en Facebook. Para la descripción de los contenidos, los participantes encontrarán cinco formularios independientes, pero con los mismos requerimientos en cada uno. Dichos requerimientos son: 1. Descripción del nombre del formato de anuncio para Facebook que desea utilizar. 2. Descripción estructural y técnica de la pieza, su formato, extensión, programa de desarrollo y herramientas necesarias para la construcción del contenido propuesto por el grupo. 3. Descripción de los atributos conceptuales o comunicativos. Una vez completados los requerimientos, los participantes debían (a través de la plataforma), enviar las respuestas para su organización, tabulación estadística y análisis. Finalmente, a los participantes se les indagó sobre el conocimiento que tuvieran de los formatos de anuncio aptos para Facebook.

\section{RESULTADOS}

El total de participantes del ejercicio fue de 70 individuos, divididos en 14 grupos (dos grupos por cada institución universitaria). Al indagar en cada uno de los grupos sobre el conocimiento de los formatos de anuncio para la red social Facebook, se descubrió que, de los 22 tipos de formatos, los participantes conocen, han escuchado o han interactuado con un total de 15 de ellos. De la misma manera, el ejercicio evidencia que, de los 15 formatos conocidos por los estudiantes, 13 fueron propuestos durante la actividad.

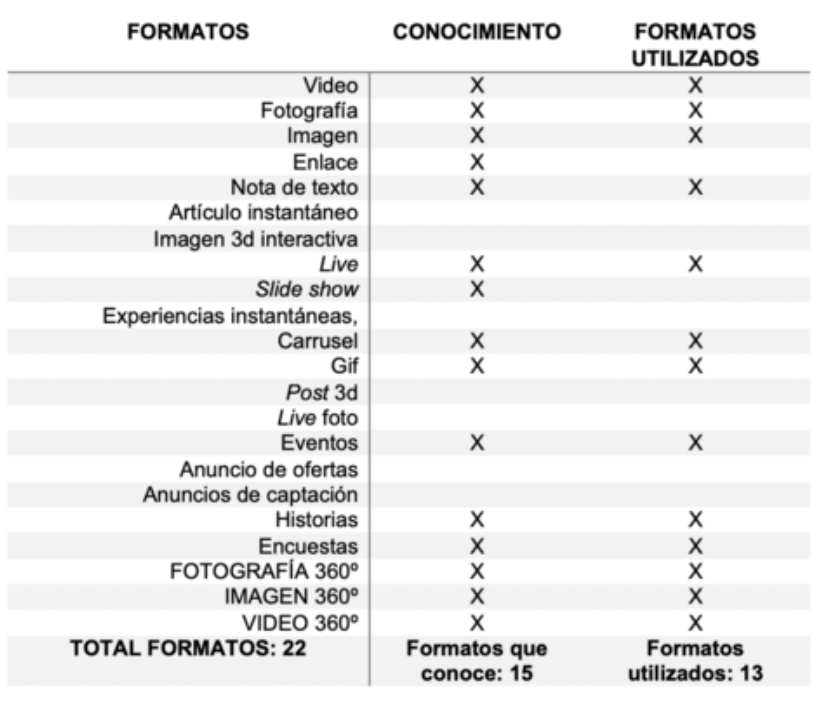

Tabla 2.

Resumen de resultados del ejercicio desarrollado por los estudiantes. Fuente: elaboración propia, 2019. 
El número total de propuestas hechas por los participantes fue de 70 . De las propuestas presentadas, tres no corresponden a un formato de anuncio válido o aceptado por Facebook.

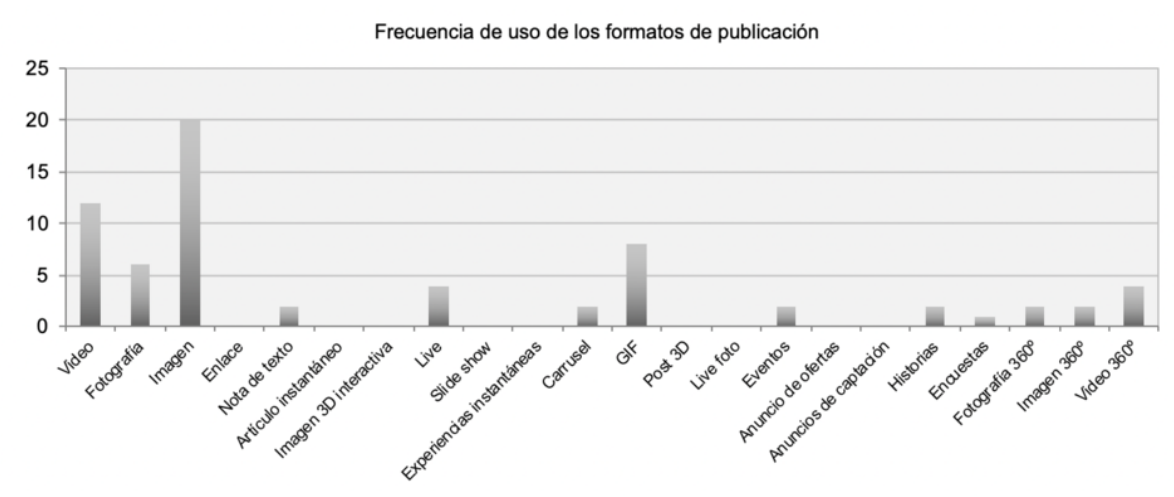

Gráfico 1 .

Frecuencia de uso de los formatos de publicación Fuente: elaboración propia, 2019.

El $57,1 \%$ de las propuestas realizadas se concentraron en los tres formatos con mayor número de menciones: imagen, con un total de 20 menciones, seguido por el video, con 12 menciones y, en tercer lugar, el formato GIF, con un total de 8 menciones. $\mathrm{Al}$ indagar a los participantes sobre las razones por las cuales sienten afinidad con los tres formatos más comunes propuestos, mencionan que estos son familiares, debido a que se desarrollan en los programas Adobe Illustrator, Adobe Photoshop, Movie-Maker, iMovie y, en menor medida, Adobe Premiere, que son aprendidos durante su desarrollo educativo profesional.

Al consultar a los participantes durante el ejercicio, se evidenció que desconocen el $31,8 \%$ de los formatos textuales o multimedia que Facebook permite publicar como alternativa para el desarrollo de contenidos. El 40,9\% de los formatos de anuncio de Facebook no fueron tenidos en cuenta durante el ejercicio, según la descripción de los participantes; estos formatos no fueron mencionados debido al desconocimiento en su elaboración y publicación.

Nueve grupos de los dieciséis participantes decidieron repetir un tipo de formato de anuncio, argumentando desconocimiento de los demás formatos. De los dieciséis grupos participantes, siete decidieron abstenerse de realizar una propuesta, debido a que sus integrantes no sabían describir el nombre o desarrollo del formato que deseaban proponer.

Del $100 \%$ de las propuestas recibidas, el 5,7\% menciona el formato $360^{\circ}$ como opción para el desarrollo de contenidos del ejercicio propuesto. Del $100 \%$ de las propuestas recibidas, el $1,4 \%$ conoce el formato "Video $360^{\circ}$ " y, adicionalmente, tiene conocimientos sobre su grabación, edición, pero no sobre su publicación. Uno de los dieciséis grupos participantes tiene conocimiento sobre el proceso de desarrollo, edición y publicación de videos $360^{\circ}$. Las cuatro propuestas realizadas bajo el formato video $360^{\circ}$ describen una experiencia de inmersión entre los espectadores y el espacio físico de la marca. Se evidenció confusión en 
la mayoría de los grupos al discutir si un video $360^{\circ}$ se clasifica dentro de la misma categoría con la fotografía $360^{\circ}$ y la imagen $360^{\circ}$.

Quince de los dieciséis grupos participantes mencionaron que el responsable de la construcción de contenidos para las marcas es el community manager. Uno de los grupos participantes mencionó que el responsable de la creación de contenidos es el content manager.

El 87,1\% de las propuestas mencionadas por los participantes están ligadas a la presentación interna del espacio físico de la bolera, pero en su mayoría se proponen con formatos que no permiten evidenciar esta acción.

\section{DISCUSIÓN}

El continuo avance y transformación de las plataformas digitales nos obliga a mantener una postura de actualización para el desarrollo de contenidos que trasciendan el simple objetivo de comunicar, y lleguen al campo de la interacción y la generación de engagement. Esta característica aporta en el desarrollo de una percepción positiva de marca y se logra a partir del conocimiento de las acciones que intervienen en el desarrollo táctico de las campañas publicitarias en internet. Los futuros creadores de contenidos para la plataforma Facebook deben contar con competencias de desarrollo y conocimiento de todo tipo de herramientas que nos brindan los mismos espacios de anuncio para la comunicación de mensajes.

Internet se perfila como un espacio potencial de desarrollo para la comunicación publicitaria, por ello es imperativo desarrollar competencias que les permitan a los profesionales de Publicidad, Comunicación y Diseño gráfico generar engagement con los espectadores o seguidores a partir del correcto uso de los nuevos medios y canales para la divulgación de mensajes.

Se evidencia el desconocimiento de las herramientas y habilidades con las que debe contar un creador de contenidos o responsable del desarrollo de piezas comunicativas en los nuevos medios como las plataformas digitales, por lo cual es necesario profundizar en el conocimiento de la Publicidad Digital y la Nueva Publicidad Digital descrita por Carrillo y Castillo (2005), con el objetivo de marcar una diferencia entre una pieza multimedia con bajo contenido de interacción y una pieza que combine las tres características de la interactividad (control activo, sincronía y multidireccionalidad).

Solamente en doce de las setenta descripciones de contenidos se evidenció una propuesta que cumpliera con el contexto de interactividad propuesta por Carrillo y Castillo (2005), donde se define una acción participativa que vaya más allá de activar la pieza multimedia mediante un clic, lo que sugiere una posibilidad de profundización en los espacios académicos con respecto a la definición de interactividad y cómo desarrollar contenidos multimedia para generar engagement con los seguidores. 
Durante el desarrollo del ejercicio, la mayoría de los participantes dieron solución al problema, mencionando los formatos más comunes que, por sus características, no cuentan con un alto nivel de interactividad; por ende se reduce la opción de generar engagement entre la marca y sus seguidores, lo que traduce según las autoras Viñarás-Abad y CaerolsMateo (2016, p.171), en deficiencia de conexión emocional con la marca. Estos resultados evidencian las oportunidades de profundización en el desarrollo de programas de educación profesional que enriquezcan el conocimiento en la elaboración de contenidos multimedia para el espacio digital y que cumplan con el objetivo de generar engagement entre los espectadores y las marcas. El contexto sobre los formatos más utilizados por los estudiantes, presentado durante la fase de resultados de la investigación, demuestra que estos individuos hacen correcto uso de las herramientas y conocimientos otorgados en las instituciones universitarias, razón por la cual ahondar en la enseñanza de nuevas tecnologías de información multimedia permitirá a los futuros creadores de contenidos desarrollar competencias de comunicación interactiva para cumplir el objetivo de divulgación de mensajes y recordación en las plataformas sociales de las marcas.

El esfuerzo por la comprensión y el desarrollo de la publicidad en los medios web debe continuar a partir de nuevas investigaciones que den cumplimiento con el objetivo de reducir el desconocimiento de herramientas, estrategias, tácticas y metodologías que permitan ejercer un trabajo eficiente y eficaz en favor del desarrollo social y de la innovación.

El formato de video $360^{\circ}$ cuenta con la característica de ser un producto con alto nivel de interacción, sin embargo, el conocimiento que se tiene sobre su desarrollo, edición y ejecución es mínimo; por ello se hace necesario proyectar espacios educativos que les permitan a los estudiantes identificar, saber manejar y publicar este tipo de piezas multimedia, que generan alto nivel de interacción y engagement.

Los estudiantes que conocen o han interactuado con el video $360^{\circ}$ son conscientes del alto nivel de interacción que se genera a partir de la inmersión que una persona puede hacer mediante este tipo de tecnología. Los hallazgos y propuestas de profundización serán comunicadas mediante charlas con las universidades involucradas en el ejercicio de participación, cumpliendo el compromiso pactado para el desarrollo de dicha investigación y buscando cumplir con el objetivo de presentar y promover el uso de herramientas de publicación en la red social de mayor uso en el mundo.

\section{REFERENCIAS}

Adán, P., Arancibia, R., López, A., Ramírez, J., Sospedra, R., \& Valladares, Á. (2016). Business to Social, Marketing digital para empresas y personas (Primera edición ed). Madrid, España: Alfaomega.

Aguirre, C., \& Odriozola, J. (2017). Más allá de la interactividad: uso de herramientas interactivas en cibermedios ecuatorianos. Combumanitas: Revista cientifica de comunicación, 8(2), 58-72. 
Ángel, M., \& Martínez, E. (2014). Comunicación empresarial en redes sociales: gestión de contenidos y experiencias. Methaodos. Revista de ciencias sociales, 2(2), 276-285.

Arroyo, I., Calle, S., \& Van Wyk, C. (2018). La eficacia en la comunicación de las ONGD. El uso de Facebook en campañas de emergencia. RLCS, Revista Latina de Comunicación Social, 73(7), 765 - 789.

Barker, M., Barker, D., Bormann, N., \& Neher, K. (2013). Marketing para redes sociales. Cengage Learning Editores, S.A. de C.V.

Barba Abad Magdalena; Alvarado Nando, Maritza; De La Rosa Daza, Deisy; Mora Ramírez, Ángela Julieta, M. B. M. (2019). Innovación educativa, nuevas metodologías y experiencias en el aula, experiencias desde México y Colombia (1st ed.). Bogotá: Institución Universitaria Politécnico Grancolombiano.

Botella, C., García-Palacios, A., Baños, R., \& Quero, S. (2007). Realidad virtual y tratamientos psicológicos. Medicina psicosomática y Psiquiatría de enlace (82), 17-31.

Carrillo, M. (2004). La interactividad: un reto para la publicidad en el entorno digital online. ZER - Revista de Estudios de Comunicación (18), 9-24.

Carrillo, V., \& Castillo, A. (2005). La Nueva Publicidad Digital (NPD): servicios digitales y contenidos interactivos que generen 'experiencias' en los consumidores. Razón y Palabra, 10(45), 2.

Castelló, A., del Pino, C., \& Tur-Viñes, V. (2015). Estrategias de contenido con famosos en marcas dirigidas a público adolescente. (F. García, \& M. Gertrudix, Edits.). Icono, 14(1), 123-154.

Daza-Orozco, CE (2019). Iniciación científica: conceptualización, metodologías y buenas prácticas (1st ed.; C. E. Daza-Orozco, ed.). Bogotá. Politécnico Grancolombiano. https://doi.org/10.13140/RG.2.2.20812.2 3684

Daza-Orozco, CE. (2015). Investigación y emprendimiento: experiencias de las Instituciones de Educación Superior Mesa IEST (Mesa IEST (ed.)). Corporación Internacional para el Desarrollo Educativo. https://doi.org /10.13140/RG.2.1.4728.3283

De Salas, I. (2010). La publicidad en las redes sociales: de lo invasivo a lo consentido. Icono, 14(15), 75-84.

Facebook Business. (s.f.). Formatos de publicaciones que pueden incluir contenido de marca. Recuperado el 5 de 10 de 2018, de Facebook para empresas: htt ps://www.facebook.com/business/help/759742620834346

Fernández, E., \& Díaz,J. (2015). La estrategia de marketing del sector del juguete en Facebook: análisis de la campaña de navidad 2014 en España. Prisma Social Revista de Ciencias Sociales, (14), 124-151.

Gallardo, J., \& Lavin, E. (2015). El vídeo esférico en YouTube y su influencia en el contenido audiovisual. Opción, 31(4), 460-480.

Garrigo, P. (2006). ¿Hacia dónde va la publicidad en internet? Del banner al clic to call. Icono, 14(6), 99-112.

Habig, J. (2016). Think With Google. (Google, Editor). Recuperado de: https://www.thinkwithgoogle.com/intl/es-419/recursos-y-herramie ntas/video/360-video-advertising/

Hernández, R., Fernández, C., \& Baptista, P. (2010). Metodología de la investigación. México: McGraw-Hill. 
Martín, V. (2015). Social Media, herramientas de gestión para el community manager. Madrid, España: FC Editorial, Laborprex Editores.

Martínez-Rodrigo, E., \& Sánchez-Martín, L. (2011). Publicidad en internet: nuevas vinculaciones en las redes sociales. Revista de Comunicación Vivat Academia, (117E), 469-480.

Méndiz, A. (2010). Advergaming: concepto, tipología, estrategias y evolución histórica. Icono 14(15), 37-58.

Mir, P. (2016). Brand.com. (A. García Reyes, Ed.). Pamplona, España: ECOE Ediciones.

Mir, P., Fondevila, J., \& Gutiérrez, Ó. (2006). El uso de las brand fan pages de Facebook entre jóvenes y profesionales: análisis empírico. (U. d. Zulia, Ed.). Enlace Revista Venezolana de Información, 13(1), 28-44.

Moro, M., \& Rodés, A. (2014). Marketing Digital. (M. López Raso, Ed.) Madrid, España: Paraninfo S.A.

Muela, C. (2008). La publicidad en Internet: situación actual y tendencias en la comunicación con el consumidor. ZER - Revista de Estudios de Comunicación, 13(24), 183-201.

Naranjo, L. (2016). Del video en 360 grados a la realidad virtual: guia práctica para grabar un video en 360. Madrid, España: Universidad Complutense de Madrid.

Noguera, J., Martínez, J., \& Grandío, M. (2011). Redes sociales para estudiantes de Comunicación. Barcelona, España: Editorial UOC.

Norman-Acevedo, E. (2018). Rompiendo Barreras: 10 Años de la Educación Virtual en el Politécnico Grancolombiano. (primera; Eduardo NormanAcevedo, ed.). Retrieved from http://alejandria.poligran.edu.co/handle/ $10823 / 1146$

Norman-Acevedo, Eduardo. (2018). Rompiendo Barreras: 10 Años de la Educación Virtual en el Politécnico Grancolombiano. (primera; Eduardo Norman-Acevedo, ed.). Bogotá: Institución Universitaria Politécnico Grancolombiano.

Norman-Acevedo, Eduardo. (2019). NUEVOS LENGUAJES PARA APRENDIZAJE VIRTUAL HERRAMIENTAS PARA LOS ESCENARIOS DE APRENDIZAJE. PANORAMA, 13(24), 5. https:// doi.org/10.15765/pnrm.v13i24.1214

Núñez, P., García, A., \& Abuín, N. (2013). Profesionales digitales en publicidad y comunicación. Una aproximación a las necesidades del mercado laboral. CIC Cuadernos de Información y Comunicación, 18, 177-187.

Pérez, S., Melle, M., \& Paniagua, F. (2018). Innovación en radiotelevisiones públicas europeas: narrativas inmersivas y organización de los contenidos 360 grados en plataformas digitales. RLCS, Revista Latina de Comunicación Social, 73 (9), 1115-1136.

Romero, M., \& Fanjul, C. (2010). La publicidad en la era digital: el microsite como factor estratégico de las campañas publicitarias online. Revista Cientifica de Educomunicación, 17(34), 125-134.

Rowles, D. (2014). Digital Branding. (E. Ediciones, Ed.) New York, Estados Unidos: TEELL Editorial.

Sánchez, M. (2017). La influencia de las redes sociales virtuales en la difusión de información y conocimiento: estudio de pymes. (F. A. Serra, Ed.). Revista Ibero Americana de Estratégia-RIAE, 16(4), 23. 
Selva, D., \& Martín, P. (2019). Realidad virtual, publicidad y menores de edad: otro reto de la cibersociedad ante las tecnologías inmersivas. Icono, 17(1), 83-110.

Valerio, G., Herrera, D., Villanueva, F., Herrera, N., \& Rodríguez, M. (2015). Relación entre los formatos de publicación y el engagement digital: estudio de las páginas de Facebook de las universidades mexicanas. Revista de Universidady Sociedad del Conocimiento, 12(1), 50-64.

Velandia Vargas, G. K., Malangón Torres, M. A., Ramos Duarte, N., Quiroz Rubiano, M. M., Rodríguez Rubio, A., \& Celemín Pabón, Y. A. (2019). Experiencias de innovación educativa - Tomo 2 (1st ed.; L. M. TrujilloFlórez, ed.). Bogotá: Politécnico Grancolombiano.

Viñarás, M., \& Caerols, R. (2016). \#5Museos: un caso de éxito sobre la oportunidad de las redes sociales para generar engagement. Revista Internacional de Relaciones Públicas, 6(12), 169-190.

Zarrella, D., \& Zarrella, A. (2011). Marketing con Facebook. (V. Ruiz Calderon, E. García, Eds., \& A. Rubio Orraca, Trad.) Madrid, España: Ediciones Anaya Multimedia.

\section{Información adicional}

Para citar este articulo: / To cite this article: / Para citar este artigo:: ParraMayorga, R. (2020). INVESTIGACIÓN EN UNIVERSIDADES DE BOGOTÁ SOBRE EL CONOCIMIENTO Y DESTREZA DE LOS FUTUROS CREADORES DE CONTENIDO DIGITAL PARA LA CONSTRUCCIÓN DE FORMATOS DE ANUNCIO EN FACEBOOK. (Politécnico Grancolombiano, Ed.). Revista Panorama, 14(27), DOI: http://dx.doi.org/10.15765/pnrm.v14i27.1529 